\title{
Fatty acid composition and cpDNA content in Arabidopsis thaliana mutants deprived of EGY1 protease
}

\author{
M. ADAMIEC ${ }^{*,+}$ (D) , M. SZOMEK ${ }^{* *}$, E. GABAŁA ${ }^{* * *}$, J. DOBROGOJSKI $^{\sharp}$, L. MISZTAL \\ and R. LUCIŃSKI*
}

Adam Mickiewicz University, Faculty of Biology, Institute of Experimental Biology, Department of Plant Physiology, Umultowska 89, 61-614 Poznań, Poland*

Department of Biochemistry and Molecular Biology, University of Southern Denmark, Campusvej 55, M 5230 Odense, Denmark**

Institute of Plant Protection, National Research Institute, Wegorka 20, 60-318 Poznań, Poland ${ }^{* * *}$

Department of Biochemistry and Biotechnology, Faculty of Agronomy and Bioengineering, Poznan University

of Life Sciences, Dojazd 11, 60-637 Poznań, Poland\#

\begin{abstract}
EGY1 (ethylene-dependent gravitropism-deficient and yellow-green 1) is an intramembrane metalloprotease located in chloroplasts, involved in many diverse processes including chloroplast development, chlorophyll biosynthesis, and the ethylene-dependent gravitropic response. Plants deprived of this protease display pleiotropic effects such as the yellow-green early senescence phenotype and a poorly developed thylakoid system membrane in the mature chloroplasts. We applied the GC/MS technique to analyze the changes in fatty acid composition in two egyl mutant lines. We used DAPI staining and transmission electron microscopy methods to establish the number of nucleoids and the amount of chloroplast DNA. Our results indicated that the lack of EGY1 protease led to a dramatic overaccumulation and a dramatic decrease in the content of linolenic acid C18:3 and hexadecatrienoic acid C16:3, respectively. The amount of chloroplast DNA and the number of nucleoids were severely reduced in egyl mutant lines. Similarly, a reduced correlation between DAPI and autofluorescence signal was observed, which may indicate some perturbations in nucleoid anchoring.
\end{abstract}

Keywords: Arabidopsis thaliana; chloroplasts; EGY1; fatty acids; nucleoids.

\section{Introduction}

EGY1 is a chloroplast intramembrane metalloprotease crucial for many aspects of the chloroplast functions. The lack of the protease leads to pleiotropic effects including the early senescence phenotype, deficiency in ethylene- induced gravitropism, hypersensitivity to ammonium, and resistance to phosphate starvation (Yu et al. 2016, Adamiec et al. 2017). The hypocotyls of plants deprived of the protease were characterized by a significant decrease in glucose and starch content. Additionally, lower content of fatty acids and a decrease in the $\mathrm{C} 18$ : $\mathrm{C} 16$ ratio were

\section{Highlights}

- EGY1 protease is involved in the regulation of unsaturated fatty acid composition

- Lack of EGY1 protease leads to overaccumulation of linolenic acid

- The number of nucleoids in chloroplasts of egyl mutants is severely reduced
Received 4 August 2021

Accepted 2 November 2021

Published online 25 November 2021

${ }^{+}$Corresponding author

e-mail:msolin@amu.edu.pl

Abbreviations: cpDNA - chloroplast DNA, EGY1 - ethylene-dependent gravitropism-deficient and yellow-green 1, TEM - transmission electron microscopy, WT - wild type.

Acknowledgements: This work was supported by the National Science Centre, Poland based on the decision number DEC-2014/15/B/ $\mathrm{NZ3/00412.}$

Conflict of interest: The authors declare that they have no conflict of interest. 
observed, and it was suggested that EGY1 may be involved in fatty acid elongation (Guo et al. 2008). The mature leaves of egyl Arabidopsis thaliana mutants display a yellow-green phenotype due to lower chlorophyll (Chl) content and are characterized by shortened leaf longevity. Furthermore, in the mature chloroplasts of egyl mutants, reduced grana thylakoids and poorly developed lamellae networks were observed. The soluble protein contents and ion leakage in egyl Arabidopsis thaliana leaves were also investigated. Ten days after leaf emergence, both the soluble protein content and the ion leakage were similar in egyl and WT plants. Then, an increase was observed in ion leakage and a decrease in soluble protein content (Chen et al. 2016). The EGY1 protease is involved in lipid biosynthesis (Guo et al. 2008) and it cannot be excluded that observed disturbances in chloroplast biogenesis, especially underdevelopment of the lamella network, are a result of impaired lipid homeostasis. The chloroplast thylakoid membrane system is an important structure not only for photosynthetic electron transport chain but also for nucleoid anchoring. In mature wild-type plants, a substantial number of nucleoids is associated with chloroplasts' thylakoid membranes (Sakamoto and Takami 2018). Thylakoid membrane binding was suggested to play an important role for cpDNA replication and active gene expression necessary for maintaining the functionality of photosynthetic structures (Sakai et al. 2004, Oldenburg and Bendich 2015). There is, however, no information concerning the number of nucleoids in egyl chloroplasts. There is also no information about the lipid composition of egyl mature leaves. We decided to investigate this issue.

\section{Material and methods}

Plant material and growth conditions: Previously described (Chen et al. 2005) Arabidopsis thaliana mutant lines carrying the T-DNA insertion in AT5G35220 were applied to investigate the effects of the absence of EGY1 protease on fatty acid composition and amount of cpDNA. Namely, SALK_134931 (described as egy1-2) and SALK 061494 (described as egy1-3) were used. The seeds were obtained from NASC (Nottingham Arabidopsis Stock Centre, Nottingham, UK).

Wild-type Arabidopsis thaliana (L.) Heynh (ecotype Columbia) (WT), as well as egyl-2 and egy1-3 mutant lines, were grown on sphagnum peat moss and wood pulp (Jiffy peat pellets, AgroWit, Przylep, Poland) under a 16-h light/8-h darkness photoperiod, at an irradiance of $110 \mu \mathrm{mol}$ (photon) $\mathrm{m}^{-2} \mathrm{~s}^{-1}$ (white fluorescent light lamp Philips Master T-E-D 58 W/840 REFLEX Eco), constant temperature of $22^{\circ} \mathrm{C}$, and relative humidity of $70 \%$.

Homozygosity of the mutants: The homozygosity of used mutant lines was previously demonstrated (Chen et al. 2005, Adamiec et al. 2021). The absence of the EGY1 protease protein was additionally confirmed by us with the standard Western blot procedure (Adamiec et al. 2018). The total leaf protein used in the procedure was isolated using the Protein Extraction Buffer (PEB, Agrisera, Vännäs, Sweden) according to the producers' protocol.
The specific Anti-EGY1 polyclonal antibodies were exclusively produced in rabbits by Agrisera $A B$ (Vännäs, Sweden) using the highly purified N-terminal region (aa 50-250) of EGY1 protein from $A$. thaliana. The AntiLhcb5 antibody was purchased from Agrisera AB (Vännäs, Sweden).

Preparation of leaf samples for transmission electron microscopy: For the transmission electron microscopy analysis, the fifth leaf from four-week-old plants was used. Leaf samples $(5 \times 5 \mathrm{~mm})$ derived from both Arabidopsis thaliana wild-type ecotype Columbia 0 (Col-0) and egy 1 mutants of Col- 0 background, were fixed in a mixture of $4 \%$ glutaraldehyde and $4 \%$ paraformaldehyde $(1 / 1$; v/v) overnight at $4^{\circ} \mathrm{C}$ (Morris and Karnovsky 1965). The samples were rinsed three times for 15 min with $0.1 \mathrm{M}$ cacodylate buffer. Post-fixation was conducted in osmium tetroxide $\left(\mathrm{OsO}_{4}\right)$ for $24 \mathrm{~h}$ at $4^{\circ} \mathrm{C}$. Fixed samples were stained in a $1 \%$ aqueous solution of uranyl acetate. Subsequently, dehydration was performed in a series of ethanol solutions. Eventually, leaf samples were embedded in low-viscosity Spurr's epoxy resin (Spurr 1969) and observed under an $H T 7700$ transmission electron microscope (Hitachi, Tokyo, Japan) (Borek et al. 2006).

Preparation of chloroplasts for visualization of nucleoids: The $A$. thaliana leaves were harvested after $8 \mathrm{~h}$ of darkness. All steps were performed in the cold room, under the green light, as previously described (Adamiec et al. 2020). A. thaliana leaves (0.2 g) were homogenized briefly in $2 \mathrm{~mL}$ of an ice-cold homogenized buffer [50 mM HEPES-KOH, pH 7.8, $330 \mathrm{mM}$ sorbitol, $10 \mathrm{mM}$ EDTA, $5 \mathrm{mM} \mathrm{NaCl}, 5 \mathrm{mM} \mathrm{MgCl}_{2}, 5 \mathrm{mM}$ sodium ascorbate, and $0.2 \%(\mathrm{w} / \mathrm{v}) \mathrm{BSA}]$. The homogenate was transferred to $40 \%$ Percoll and the sample was centrifuged for $6 \mathrm{~min}, 1,700 \times \mathrm{g}$ at $4^{\circ} \mathrm{C}$. Isolated chloroplasts were attached to the $35 \mathrm{~mm}$ corning dish using a poly-D-lysine coating $\left(0.1 \mathrm{mg} \mathrm{mL}^{-1}\right)$ and were allowed to settle for $1 \mathrm{~h}$ and washed with $1 \mathrm{~mL}$ of PBS to remove nonadherent structures. To visualize DNA chloroplasts, they were labeled with DAPI (final concentration of $0.6 \mu \mathrm{g} \mathrm{mL}-1$ ) and observed using a confocal fluorescence microscope Nikon Ti2, A1 LFO with a Plan $100 \times$ NA 1.4 oil objective. Fluorescence from chlorophyll was excited with a $488 \mathrm{~nm}$ laser and DAPI with $405 \mathrm{~nm}$ with the Galvano scanner. The emission wavelengths were as follows: $662-737 \mathrm{~nm}$ for chlorophyll autofluorescence and 425-475 nm for DAPI (Evans et al. 2010, Adamiec et al. 2020). All the images were background corrected. From each image, chloroplasts were selected using ImageJ.

Colocalization analysis: The colocalization analysis of the chlorophyll autofluorescence and DAPI signal was measured with ImageJ with the $J a C o P$ plugin (Bolte and Cordelières 2006).

GC/MS analysis of fatty acid composition in leaves: The leaves of four-week-old Arabidopsis thaliana WT plants and egyl mutants were used. Leaf samples $(100 \mathrm{mg})$ were placed in $1 \mathrm{ml}$ of $0.5 \mathrm{M} \mathrm{NaOH}$ dissolved in methanol 
and $10 \mu \mathrm{l}$ of methanol solution of hexadecanoic acid (3.22 mg mL $\mathrm{mL}^{-1}$ ) as internal standard was added. The samples were incubated for $10 \mathrm{~min}$ at $90^{\circ} \mathrm{C}$. Next $2 \mathrm{ml}$ of $12 \%(\mathrm{w} / \mathrm{v}) \mathrm{BF}_{3}$ in methanol was added and the samples were incubated for another $2 \mathrm{~h}$ at $90^{\circ} \mathrm{C}$. After this time, $200 \mu \mathrm{l}$ of $\mathrm{NaOH}$ saturated solution in methanol was added. Fatty acid methyl esters (FAME) were extracted with $1 \mathrm{ml}$ of hexane and the hexane solution containing FAME was subjected to GC/MS analysis. The GC/MS analysis was performed with a GCMS-QP2010 Ultra gas chromatograph (Shimadzu, Kyoto, Japan) connected to a QP-5000 quadrupole mass spectrometer (Shimadzu, Kyoto, Japan). The GC/MS was equipped with an AOC-5000 autosampler (Shimadzu, Kyoto, Japan). The samples were separated by a ZB-FAME (Agilent Technology, Santa Clara, USA) capillary column: $(30 \mathrm{~m} \times$ $0.25 \mathrm{~mm}, 0.20 \mu \mathrm{m}$ stationary phase). The carrier gas $(\mathrm{He})$ flow was $11.9 \mathrm{ml} \mathrm{min}^{-1}$, the partition coefficient was 10 , and the pressure program was adjusted to the temperature program. The analysis conditions were as follows: dispenser temperature of $250^{\circ} \mathrm{C}$, ion source and transfer line temperature: $250^{\circ} \mathrm{C}$. The following temperature program was used: initially, $100^{\circ} \mathrm{C}$ held for $2 \mathrm{~min}$, then $10^{\circ} \mathrm{C}$ per min to $140^{\circ} \mathrm{C}$, held for $5 \mathrm{~min}$, then $3^{\circ} \mathrm{C}$ per min to $190^{\circ} \mathrm{C}$, held for $1 \mathrm{~min}$, then $20^{\circ} \mathrm{C}$ per min to $280^{\circ} \mathrm{C}$, held for $1 \mathrm{~min}$, analysis time was $37 \mathrm{~min}$. Electron ionization (EI) with an energy of $70 \mathrm{eV}$ was used, the mass spectrometer worked in the total ion current (TIC) mode in the mass range of $35-400 \mathrm{~m} / \mathrm{z}$, the solvent cut-off time was 5 min. GCMS solution 2.53 (Shimadzu, Kyoto, Japan) was used for data analysis. The obtained mass spectra were compared with the NIST 8.0 mass spectral library.

Statistical analysis: Differences in the measured fatty acids content were analyzed for statistical significance using one-way analysis of variance $(A N O V A)$. Means were regarded as significantly different at $P<0.05$.

\section{Results}

EGY1 abundance in egy1 mutant lines: Two lines of A. thaliana mutants (egyl-2 and egyl-3) were used in the experiments. Their homozygosity has been demonstrated and described in the literature previously (Chen et al. 2005, Adamiec et al. 2021). For this reason, in this study, we only checked the level of EGY1 protein in both lines. We used Lhcb5 as a positive control. From the literature information and our own experience (Adamiec et al. 2021), we know that the content of this protein in egyl mutants does not differ from its content in the wild-type plants of A. thaliana. Western-blot experiments with specific AntiEGY1 antibodies showed, as predicted, no EGY1 protein in both mutant lines (Fig. 1).

Chloroplast ultrastructure: The ultrastructure of the chloroplasts isolated from Arabidopsis thaliana wildtype plants, egyl-2, and egyl-3 mutant lines were investigated by transmission electron microscopy (TEM). Chloroplasts were isolated from plants with the first flower opened, which is described as developmental phase 6.0 according to the BBCH scale (Boyes et al. 2001). The mature chloroplasts from wild-type plants had a fully developed thylakoid membrane system and accumulated considerable amounts of starch grains, while the thylakoid membrane system in chloroplast isolated from both egyl mutant lines was dramatically underdeveloped, and considerably smaller amounts of starch grains were accumulated. Additionally, in egyl chloroplasts, more plastoglobuli were observed, which is fully consistent with previous observations by Chen et al. (2016). TEM analysis also revealed partial rupture and deformation of chloroplast envelope membranes in egyl mutants. The most important finding from our TEM analysis pertains, however, to nucleoid structures, which were less abundant in egyl mutants than that in wild-type plants. In the data obtained from the wild-type chloroplasts, the observed median of nucleoids per cross-section was 3 , while in both egyl mutant lines, the median number of nucleoids per cross-section was 1 (Fig. 2).

Chl fluorescence and cpDNA content in egyl mutant lines: The chloroplasts of WT plants and egyl-2 and egy 1-3 mutants were DAPI-stained and DNA fluorescence for individual plastids was investigated to confirm the reduced amount of cpDNA in analyzed mutants. We found that the DAPI-cpDNA signal from stained chloroplasts was significantly weaker in both analyzed mutant lines, indicating smaller cpDNA content in chloroplasts isolated from both mutant lines. Additionally, the autofluorescence of Chl was measured. Also, in this case, the signal observed in both egyl mutant lines was significantly lower (Fig. $3 A, B$ ). The correlation of DAPI and autofluorescence signal was also established. The obtained results indicate that the correlation of both fluorescence signals is significantly lower in both egyl mutant lines, indicating a reduced frequency of nucleoids near the thylakoid membranes (Fig. 3C).

Fatty acid composition: The underdevelopment of the thylakoid membrane system and deformation of chloroplast envelope membranes in egyl mutants prompted us to investigate changes in fatty acid composition in leaves of egyl mutants. Our analysis indicated that in both wild-type and egyl mutant lines the most abundant fatty acids were $\mathrm{C} 16: 0$ and $\mathrm{C} 18: 3$. The abundance of saturated acids C16:0, C18:0, C20:0, C22:0, and C24:0 was at a similar level

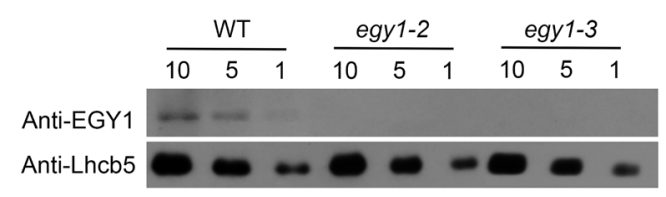

Fig. 1. Immunoblot analysis of EGY1 abundance in wildtype plants (WT), egy 1-2, and egyl-3 mutants. Samples containing 10,5 , and $1 \mu \mathrm{g}$ of total leaf protein were separated by SDS-PAGE and transferred to PVDF membranes. Subsequently the immunoblot assay with use of Anti-EGY1 and Anti-Lchb5 primary antibodies was applied. Anti-Lhcb5 was used as a positive control. 

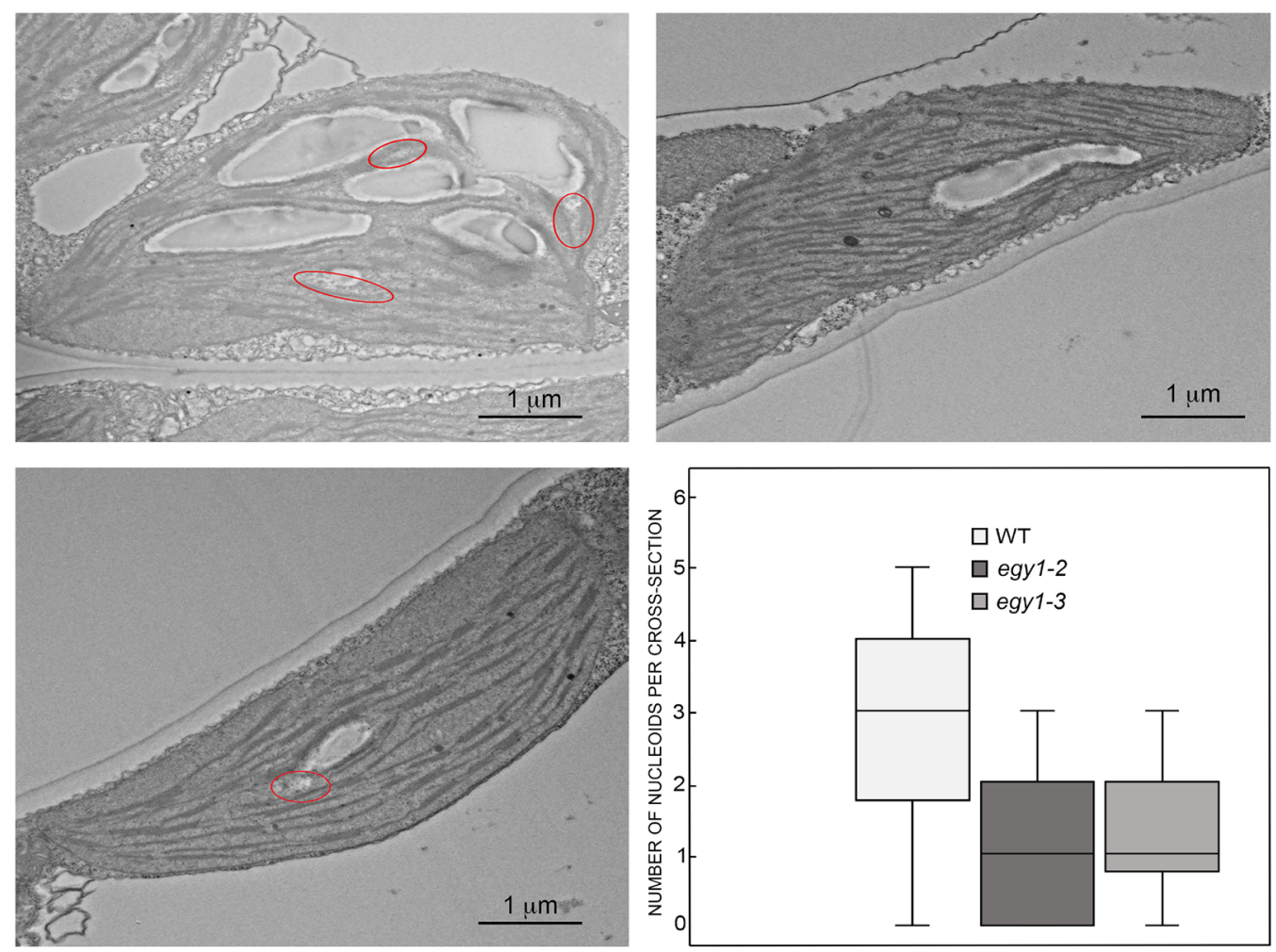

Fig. 2. Electron micrographs showing the ultrastructure of leaf chloroplasts. The nucleoid regions are marked with an arrow. Boxplot displaying distribution of nucleoid regions in investigated chloroplasts' cross-sections. The line inside the box is the median. The bottom line of the box represents the first quartile and the upper line of the box the third quartile. The whiskers represent minimum and maximum values. The boxplot was created based on data from 12 photos of chloroplasts for each analyzed plant line.

in mutants and wild-type plants. No changes were also observed in the accumulation of monounsaturated 16:1 fatty acids. However, in the content of other unsaturated fatty acids, such as $\mathrm{C} 16: 2, \mathrm{C} 16: 3, \mathrm{C} 18: 1$, and $\mathrm{C} 18: 2$, a significant decrease occurred in both egyl mutant lines. Conversely, overaccumulation of $18: 3$ was observed which consequently increased the $\mathrm{C} 18 / \mathrm{C} 16$ ratio (Table 1 , Fig. 4).

\section{Discussion}

Microscopic analysis revealed that mature chloroplasts of Arabidopsis thaliana plants deprived of EGY1 protease, apart from the underdeveloped system of thylakoid membranes, dramatically smaller starch grains and an increased number of plastoglobuli, which were previously reported by Chen et al. (2005), were also characterized by a reduced amount of chloroplast cpDNA, which has not been described before. During chloroplast differentiation, a continuous increase in cpDNA per organelle is observed from a dozen in small chloroplasts to $70-130$ copies in mature chloroplasts. However, the amount of cpDNA in chloroplasts after reaching maturity remains under discussion. Previously reported models indicated a decline in the amount of cpDNA during leaf maturity (Shaver et al. 2006, Rowan et al. 2009). Another research indicates, however, that the amount of cpDNA remains largely unchanged during senescence (Zoschke et al. 2007) and chloroplast-to-gerontoplast transition (Golczyk et al. 2014). The data concerning the number of DNA copies per chloroplast remain inconsistent due to dynamic changes during chloroplast development, significant differences between species as well as the diverse methods applied to investigate this issue (Oldenburg and Bendich 2015). The lower correlation between DAPI and autofluorescence signal indicates that the frequency of nucleoids in the proximity of thylakoid membranes is significantly lower in egyl mutants. This result may indicate some perturbations in nucleoid anchoring, which is known to be one of the important factors involved in plastid DNA replication (Powikrowska et al. 2014). The smaller fraction of nucleoids attached to thylakoid membranes may be partially the result of thylakoid membrane underdevelopment what, in turn, is related to changes in fatty acid composition, the content of which is - as suggested partly dependent on the activity of EGY1. Namely, it is suggested that the activity of EGY1 protease is associated with the elongation of $\mathrm{C} 16$ to $\mathrm{C} 18$ fatty acids (Guo et al. 2008). According to our results, the contents of saturated C18:0 and C16:0 from which the $\mathrm{C} 18$ is elongated are similar in egyl mutants and WT plants. Our analysis also revealed an increase in $\mathrm{C} 18 / \mathrm{C} 16$ ratio in both egyl mutant lines. The result is inconsistent with previous research, where the C18/C16 ratio decreased (Guo et al. 2008). 

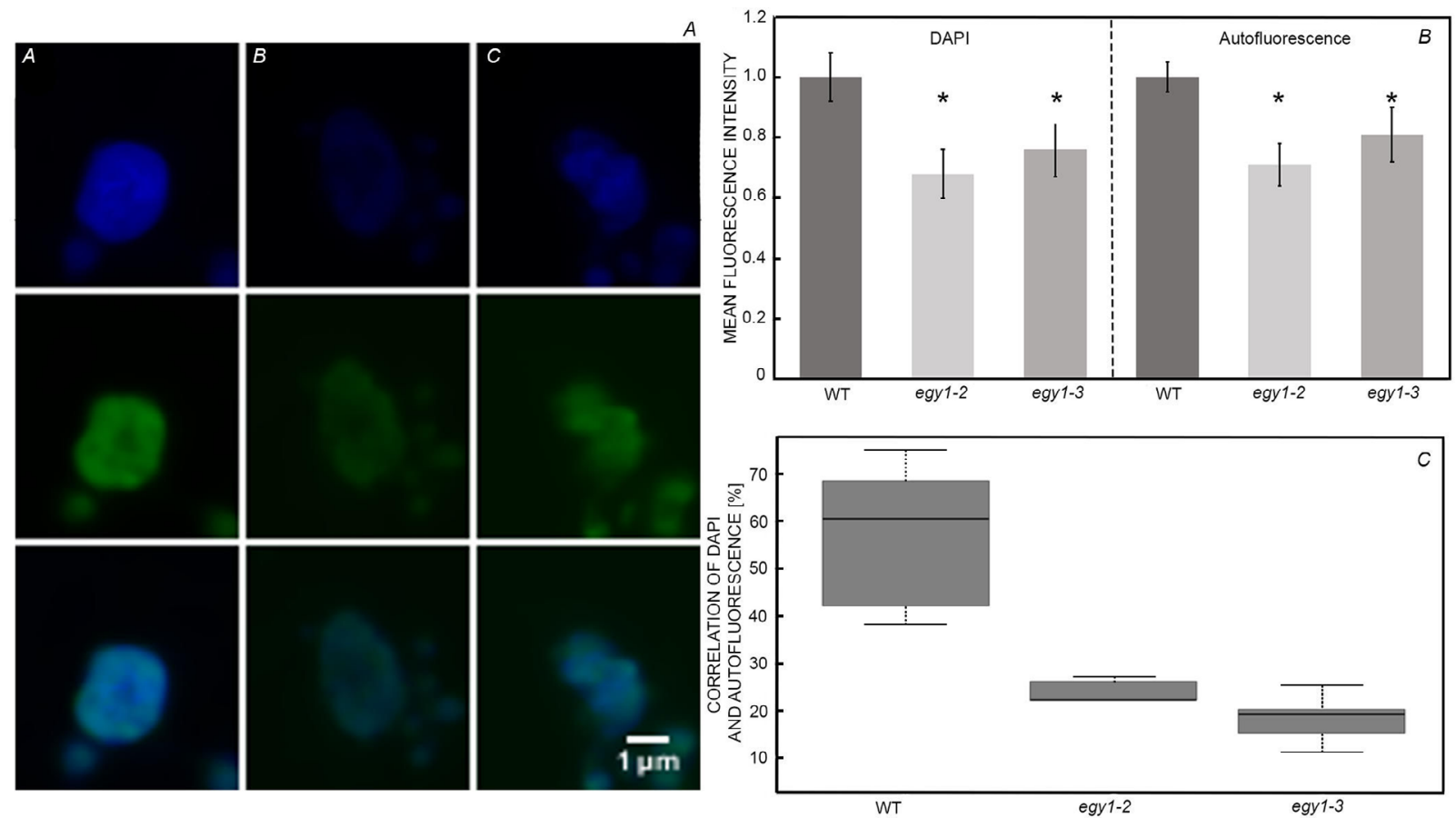

Fig. 3. Visualization of chloroplast nucleoids. The chloroplast DNA was DAPI-labeled and visualized using a confocal fluorescence microscope. $(A)$ The autofluorescence of chlorophyll (AF) was excited with 488-nm laser and the fluorescence of DAPI (DAPI) was excited with $405 \mathrm{~nm}$. $(B)$ The relative intensity of the signal from WT and mutant plants and the correlation of DAPI and autofluorescence. (C) The colocalization analysis of the chlorophyll autofluorescence and DAPI signal. The graphs were prepared based on four photos containing 20-30 chloroplast (two photos for one biological replication). The line inside the boxplot is the median. The bottom line of the box represents the first quartile and the upper line of the box the third quartile. The whiskers represent minimum and maximum values. The asterisks indicate statistically significant differences between the WT and individual mutants.

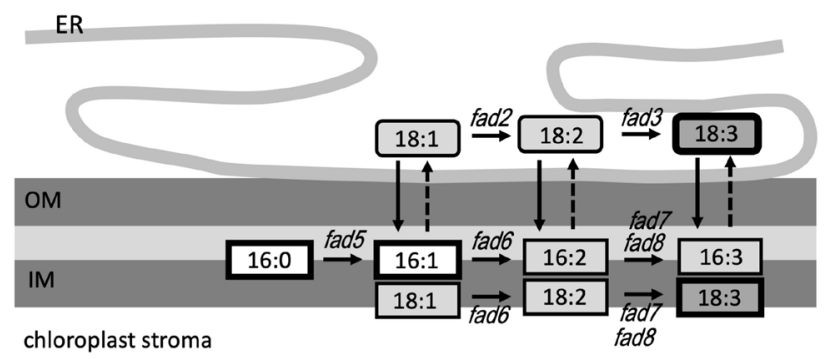

Fig. 4. The changes in fatty acid metabolism in the chloroplast and endoplasmic reticulum of Arabidopsis thaliana egyl mutant lines. The figure was created based on the model presented by Li et al. (2021). The fatty acids the content of which remains unchanged in egyl mutants are marked with the white rectangle with a bold border. The fatty acids the content of which decreases in egyl mutants are marked with a light grey and thin border and the overaccumulation fatty acids are marked with a dark gray and bold border. ER - endoplasmic reticulum; OM - outer chloroplast membrane; IM - inner chloroplast membrane.

The reason for the inconsistency is probably the fact that the analyzed plants were at different developmental stages. The decrease in C18/C16 ratio was observed in hypocotyls while our analyses were performed in mature leaves. The observed increase of $\mathrm{C} 18 / \mathrm{C} 16$ seems to be
Table 1. Relative content of fatty acids and $\mathrm{C} 18: \mathrm{C} 16$ ratio in Arabidopsis thaliana WT and egyl mutants. ' \pm ' indicates the SD determined in the analysis of samples obtained from three biological replicates. The asterisks indicate statistically significant differences between the WT and individual mutants. The statistical significance of the differences was calculated based on the one-way ANOVA, for $P<0.05$.

\begin{tabular}{lrcr}
\hline & \multicolumn{1}{l}{ WT } & \multicolumn{1}{l}{ egyl-2 } & \multicolumn{1}{l}{ egyl-3 } \\
\hline $16: 0$ & $21.61 \pm 1.54$ & $20.09 \pm 0.69$ & $20.53 \pm 0.45$ \\
$16: 1$ & $4.35 \pm 0.33$ & $4.47 \pm 0.14$ & $4.48 \pm 0.27$ \\
$16: 2$ & $0.73 \pm 0.03$ & $0.52 \pm 0.01^{*}$ & $0.50 \pm 0.01^{*}$ \\
$16: 3$ & $6.70 \pm 0.82$ & $0.91 \pm 0.01^{*}$ & $0.87 \pm 0.04^{*}$ \\
$18: 0$ & $1.98 \pm 0.27$ & $1.67 \pm 0.09$ & $1.98 \pm 0.20$ \\
$18: 1$ & $4.81 \pm 0.51$ & $2.45 \pm 0.05^{*}$ & $2.37 \pm 0.19^{*}$ \\
$18: 2$ & $20.16 \pm 0.80$ & $18.62 \pm 0.37^{*}$ & $18.57 \pm 0.59^{*}$ \\
$18: 3$ & $36.89 \pm 2.83$ & $48.88 \pm 0.90^{*}$ & $48.50 \pm 1.19^{*}$ \\
$20: 0$ & $0.19 \pm 0.00$ & $0.20 \pm 0.03$ & $0.18 \pm 0.02$ \\
$22: 0$ & $0.29 \pm 0.01$ & $0.30 \pm 0.01$ & $0.27 \pm 0.02$ \\
$24: 0$ & $0.72 \pm 0.04$ & $0.81 \pm 0.06$ & $0.75 \pm 0.04$ \\
C18:C16 ratio & $1.92 \pm 0.11$ & $2.76 \pm 0.12^{*}$ & $2.71 \pm 0.08^{*}$ \\
\hline
\end{tabular}

a result of overaccumulation of $\alpha$-linoleic acid (C18:3), which is one of the most abundant fatty acids found in A. thaliana leaves. $\alpha$-linolenic acid (C18:3) is a product of 
the desaturation of linoleic acid (C18:2) by $\omega-3$ fatty acid desaturases (FAD). In A. thaliana, two separate pathways are responsible for fatty acid desaturation. The first one is located in chloroplasts, while another is placed in the endoplasmic reticulum. The desaturation of $16: 2$ to $16: 3$ is performed exclusively in chloroplasts, in a reaction catalyzed by FAD7 and FAD8, and the accumulation level of the 16:3 fatty acid is considered as a good indicator of the activity of FAD7/8 (Afitlhile et al. 2015). Both plastid enzymes are located in the chloroplast envelope (Koo and Ohlrogge 2002, Ferro et al. 2003). Additionally, FAD7 was also found in the thylakoid membranes (Andreu et al. 2007). In turn, the desaturation of $18: 2$ to $18: 3$ may be performed in two independent pathways, in chloroplasts and the endoplasmic reticulum. The chloroplast pathway is FAD7 and FAD8 dependent, while the endoplasmic reticulum pathway is carried out by the FAD3 (Dyer and Mullen 2001). Moreover, the cases of an increased amount of $\alpha$-linoleic acid described in the literature are most often associated with increased activity of the FAD3 (Shah et al. 1997, O'Neill et al. 2011).

Taking this into account, we can conclude that the activity of FAD7/8 in egyl mutants is lower than that in WT plants and thus overaccumulation of linolenic acid is probably due to the increased activity of FAD3.

The involvement of EGY1 protease in the regulation of fatty acids biosynthesis is probably indirect and may, at least partially, result from its role in chloroplast development.

The changes in the $\mathrm{C} 18 / \mathrm{C} 16$ ratio, between $\mathrm{WT}$ and egyl mutant lines, taking place over the various developmental stages in A. thaliana, indicate the importance of EGY1 at the various stages of ontogenesis. There is no compensation for the lack of EGY1 in mutants during plant development. We note the change in the effect of its absence, manifested by the inversion of the $\mathrm{C} 18 / \mathrm{C} 16$ ratio. Based on the previous research, some suggestions can be made about the EGY1-dependent mechanism regulating the accumulation of individual fatty acids. It has to be noted that in $A$. thaliana leaves FAD7 seems to play a more important role in the desaturation of C16:2 and C18:2 fatty acids than FAD8. Moreover, FAD7, as well as FAD3, which also play a crucial role in the desaturation of $\mathrm{C} 18: 2$ fatty acid, is regulated by abscisic acid (ABA), either at the protein content level, in the case of FAD7 (Soria-García et al. 2019) or at the gene expression level, in the case of FAD3 (Matsuda et al. 2001). These observations are consistent with the results indicating that egyl mutants are characterized by disturbed ABA signaling probably resulting from a defect in a retrograde signaling pathway that integrates with ABA signaling (Li et al. 2012).

In conclusion, the smaller cpDNA content in egyl chloroplast is, at least partially, associated with impaired replication. This can be the consequence of limited anchoring sites due to underdeveloped thylakoid membranes. Disturbance of thylakoid membranes development may be caused by a defective retrograde pathway integrated with ABA signaling. The changes in the content of unsaturated fatty acids are, in turn, the result of the altered FAD3 and FAD7 activity, which may be affected both by underdeveloped thylakoid membranes and defective ABA signaling.

\section{References}

Adamiec M., Ciesielska M., Zalaś P., Luciński R.: Arabidopsis thaliana intramembrane proteases. - Acta Physiol. Plant. 39: 146, 2017.

Adamiec M., Misztal L., Ciesielska M., Luciński R.: The changes of PSII supercomplex stoichiometry in egyl mutants are related to chlorophyll $b$ deficiency. - Photosynthetica 59: 294-302, 2021.

Adamiec M., Misztal L., Kasprowicz-Maluśki A., Luciński R.: EGY3: homolog of S2P protease located in chloroplasts. Plant Biol. 22: 734-743, 2020.

Adamiec M., Misztal L., Kosicka E. et al.: Arabidopsis thaliana egy2 mutants display altered expression level of genes encoding crucial photosystem II proteins. - J. Plant Physiol. 231: $155-167,2018$.

Afitlhile M., Duffield-Duncan K., Fry M. et al.: The toc 132 toc 120 heterozygote mutant of Arabidopsis thaliana accumulates reduced levels of hexadecatrienoic acid. - Plant Physiol. Bioch. 96: 426-435, 2015.

Andreu V., Collados R., Testillano P.S. et al.: In situ molecular identification of the plastid $\omega 3$ fatty acid desaturase FAD7 from soybean: Evidence of thylakoid membrane localization. Plant Physiol. 145: 1336-1344, 2007.

Bolte S., Cordelières F.P.: A guided tour into subcellular colocalization analysis in light microscopy. - J. Microsc. 224: 213-232, 2006.

Borek S., Ratajczak W., Ratajczak L.: Ultrastructural and enzymatic research on the role of sucrose in mobilization of storage lipids in germinating yellow lupine seeds. - Plant Sci. 170: 441-452, 2006.

Boyes D.C., Zayed A.M., Ascenzi R. et al.: Growth stagebased phenotypic analysis of Arabidopsis: A model for high throughput functional genomics in plants. - Plant Cell 13: 1499-1510, 2001.

Chen C., Wang J., Zhao X.: Leaf senescence induced by EGY1 defection was partially restored by glucose in Arabidopsis thaliana. - Bot. Stud. 57: 5, 2016.

Chen G., Bi Y.R., Li N.: EGY1 encodes a membrane-associated and ATP-independent metalloprotease that is required for chloroplast development. - Plant J. 41: 364-375, 2005.

Dyer J.M., Mullen R.T.: Immunocytological localization of two plant fatty acid desaturases in the endoplasmic reticulum. FEBS Lett. 494: 44-47, 2001.

Evans I.M., Rus A.M., Belanger E.M. et al.: Dismantling of Arabidopsis thaliana mesophyll cell chloroplasts during natural leaf senescence. - Plant Biol. 12: 1-12, 2010.

Ferro M., Salvi D., Brugière S. et al.: Proteomics of the chloroplast envelope membranes from Arabidopsis thaliana.Mol. Cell. Proteomics 2: 325-345, 2003.

Golczyk H., Greiner S., Wanner G. et al.: Chloroplast DNA in mature and senescing leaves: A reappraisal. - Plant Cell 26: 847-854, 2014.

Guo D., Gao X., Li H. et al.: EGY1 plays a role in regulation of endodermal plastid size and number that are involved in ethylene-dependent gravitropism of light-grown Arabidopsis hypocotyls. - Plant Mol. Biol. 66: 345-360, 2008.

Koo A.J.K., Ohlrogge J.B.: The predicted candidates of Arabidopsis plastid inner envelope membrane proteins and their expression profiles. - Plant Physiol. 130: 823-836, 2002.

Li B., Li Q., Xiong L. et al.: Arabidopsis plastid AMOS1/EGY1 integrates abscisic acid signaling to regulate global gene 
expression response to ammonium stress. - Plant Physiol. 160: 2040-2051, 2012.

Li J., Galla A., Avila C.A. et al.: Fatty acid desaturases in the chloroplast and endoplasmic reticulum promote susceptibility to the green peach aphid Myzus persicae in Arabidopsis thaliana. - Mol. Plant Microbe. Interact. 34: 691-702, 2021.

Matsuda O., Watanabe C., Iba K.: Hormonal regulation of tissuespecific ectopic expression of an Arabidopsis endoplasmic reticulum-type $\omega-3$ fatty acid desaturase (FAD3) gene. Planta 213: 833-840, 2001.

Morris J., Karnovsky M.: A formaldehyde-glutaraldehyde fixative of high osmolality for use in electron microscopy. J. Cell Biol. 27: 137-138, 1965.

Oldenburg D.J., Bendich A.J.: DNA maintenance in plastids and mitochondria of plants. - Front. Plant Sci. 6: 883, 2015.

O'Neill C.M., Baker D., Bennett G. et al.: Two high linolenic mutants of Arabidopsis thaliana contain megabase-scale genome duplications encompassing the FAD3 locus. - Plant J. 68: 912-918, 2011.

Powikrowska M., Oetke S., Jensen P.E., Krupinska K.: Dynamic composition, shaping and organization of plastid nucleoids. Front. Plant Sci. 5: 424, 2014.

Rowan B.A., Oldenburg D.J., Bendich A.J.: A multiple-method approach reveals a declining amount of chloroplast DNA during development in Arabidopsis. - BMC Plant Biol. 9: 3, 2009.
Sakai A., Takano H., Kuroiwa T.: Organelle nuclei in higher plants: Structure, composition, function, and evolution. - Int. Rev. Cytol. 238: 59-118, 2004.

Sakamoto W., Takami T.: Chloroplast DNA dynamics: Copy number, quality control and degradation. - Plant Cell Physiol 59: 1120-1127, 2018.

Shah S., Xin Z., Browse J.: Overexpression of the FAD3 desaturase gene in a mutant of Arabidopsis. - Plant Physiol. 114: 1533-1539, 1997.

Shaver J.M., Oldenburg D.J., Bendich A.J.: Changes in chloroplast DNA during development in tobacco, Medicago truncatula, pea, and maize. - Planta 224: 72-82, 2006.

Soria-García Á., Rubio M.C., Lagunas B. et al.: Tissue distribution and specific contribution of Arabidopsis FAD7 and FAD8 plastid desaturases to the JA- and ABA-mediated cold stress or defense responses. - Plant Cell Physiol. 60: 1025-1040, 2019

Spurr A.R.: A low-viscosity epoxy resin embedding medium for electron microscopy. - J. Ultrastruct. Res. 26: 31-43, 1969.

Yu F.W., Zhu X.F., Li G.J. et al.: The chloroplast protease AMOS1/EGY1 affects phosphate homeostasis under phosphate stress. - Plant Physiol. 172: 1200-1208, 2016.

Zoschke R., Liere K., Börner T.: From seedling to mature plant: Arabidopsis plastidial genome copy number, RNA accumulation and transcription are differentially regulated during leaf development. - Plant J. 50: 710-722, 2007.

(C) The authors. This is an open access article distributed under the terms of the Creative Commons BY-NC-ND Licence. 